\title{
Efficient generation of aqueous Fe in electrocoagulation systems for low-cost arsenic removal
}

\author{
S. Müller, T. Behrends \& C.M. van Genuchten \\ Department of Earth Sciences-Geochemistry, Faculty of Geosciences, Utrecht University, Utrecht, The Netherlands
}

\begin{abstract}
Iron-electrocoagulation (Fe-EC), the electrochemical generation of Fe adsorbents in situ, is an effective treatment solution for arsenic (As) contaminated water in decentralized areas. However, extended field trials also revealed problems regarding the efficiency over long-term operation. To investigate how $\mathrm{Fe}$ generation is affected by repeated operation of Fe-EC systems, we performed laboratory experiments over a range of electrochemical and solution chemical conditions for 15-35 runs in batch mode. Our results show that Fe generation declines continuously during repeated operation under typical field conditions, resulting in a lower Fe dose than expected based on Faraday's law. In addition, we find that efficient Fe generation can be maintained in electrolytes free of oxyanions or by applying charge dosage rates $\geq 15 \mathrm{C} \mathrm{L}^{-1} \mathrm{~min}^{-1}$. Based on these results, we discuss potential strategies to maintain the efficiency of Fe-EC field systems under realistic conditions.
\end{abstract}

\section{INTRODUCTION}

Arsenic (As) contamination of drinking water is an ongoing public health disaster, especially for decentralized communities without existing water infrastructure. Iron-electrocoagulation (Fe-EC) is a promising alternative treatment method to achieve As $<10 \mu \mathrm{g} \mathrm{L}^{-1}$, which has been demonstrated in West Bengal, India. ${ }^{1}$ In Fe-EC, a steel anode is electrochemically oxidized to generate $\mathrm{Fe}^{2+}$ in solution, which is further oxidized by dissolved oxygen to form iron oxide precipitates. Arsenic is adsorbed on the surface of the in-situ formed iron particles, which can be removed by gravitational settling or filtration.

Although Fe-EC performs well under ideal laboratory conditions, the long-term operation of Fe-EC under field conditions revealed a number of issues: The As removal efficiency (the mass of As removed per charge passed for final [As] $<10 \mu \mathrm{g} \mathrm{L}^{-1}$ ) is up to five times lower than the efficiency of systems tested in the laboratory. In addition, extensive surface layers formed on the electrodes after repeated operation in the field (Amrose et al., 2013, van Genuchten et al., 2016). In this study, we track Fe production and surface layer formation in laboratory Fe-EC systems over repeated operation to identify the key parameters that impact extended Fe-EC efficiency.

\section{METHODS}

\subsection{Experiments}

Experiments were conducted in downscaled EC cells that typically consisted of two steel electrodes spaced $1 \mathrm{~cm}$ apart with a submerged anode surface area of $18 \mathrm{~cm}^{2}$ in $200 \mathrm{~mL}$ of electrolyte, stirred continuously and open to the atmosphere. In all experiments, a constant current was applied to achieve a coulombic dose of $450 \mathrm{C} \mathrm{L}^{-1}$ and the electrodes were stored open to the atmosphere between experiments. The charge dosage rate (Amrose et al., 2014) (CDR, between 4 and $54 \mathrm{CL}^{-1} \mathrm{~min}^{-1}$ ), $\mathrm{Fe}$ anode purity (lab electrodes $=99 \% \mathrm{Fe}$, field electrodes $=92 \% \mathrm{Fe})$, and electrolyte composition (synthetic groundwater (SGW) with several modifications) were investigated over 15-35 runs.

\subsection{Analysis}

The anodic interface potential and cell voltage was measured during each experiment and the electrolyte conductivity, $\mathrm{pH}$, and oxygen concentration were measured before and after each experiment. The total concentration of $\mathrm{Fe}$ in the bulk electrolyte was determined using a modified SMWW protocol (phenanthroline method) (APHA, 2015). Using the electrochemical operating parameters, we calculated the Faradaic efficiency $F E$ for each experiment, which is the measured $\mathrm{Fe}$ concentration normalized by the Fe concentration based on Faraday's law.

$$
[\mathrm{Fe}]_{\text {faraday }}=\mathrm{I} * \mathrm{t} *(\mathrm{n} * \mathrm{~F} * \mathrm{~V})^{-1}
$$

$\mathrm{n}=2$ for Fe(II); Faraday's constant $\mathrm{F}=96485 \mathrm{C} \mathrm{mol}^{-1}$

Electrode surface layers were mechanically removed only after the last run and weighed.

\section{RESULTS AND DISCUSSION}

\subsection{Faradaic efficiency}

Figure 1 shows the $F E$ over 20 runs for duplicate experiments with high and low purity anodes 


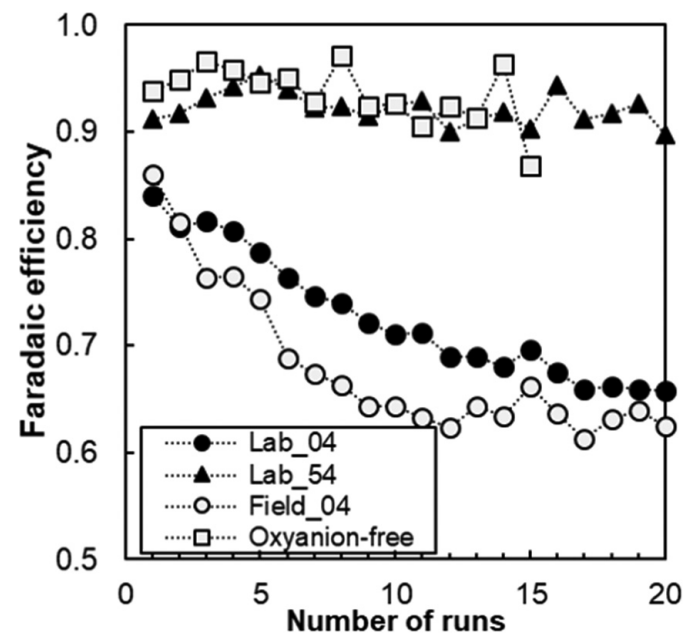

Figure 1. Faradaic efficiency $(F E)$ as a function of the number of runs of the experiment with lab electrodes at $C D R$ of $4^{\circ} \mathrm{C} \mathrm{L}^{-1} \mathrm{~min}^{-1}$ in SGW (Lab_04). Compared to Lab_04, experiments were performed in a modified electrolyte (Oxyanion-free), at higher charge dosage rate (Lab_54), or with a low purity anode instead of high purity anodes (Field_04).

(Lab_04 \& Field_04) at a CDR of $4 \mathrm{C} \mathrm{L}^{-1} \mathrm{~min}^{-1}$ in SGW. The FE of Lab_04 and Field_04 started below 1 and decreased over time, i.e. less Fe was present in the bulk solution than expected based on Faraday's law. Starting at a $F E$ of 0.85 ( $85 \%$ of the charge was converted to $\mathrm{Fe}$ in the bulk solution), the laboratory anodes showed a gradual reduction in $F E$ to 0.66 over 20 runs, which includes electrolysis and storage open to the atmosphere overnight between runs. The $F E$ of the field anode over time was similar to the laboratory anodes, decreasing to 0.62 after 20 runs, which is equal to a final $\mathrm{Fe}$ dose of approximately $80 \mathrm{mg} \mathrm{L}^{-1}$. The similarity in $F E$ between the field and laboratory electrodes suggests that a high purity Fe electrode does not necessarily translate to sustained high $F E$ at the investigated $C D R$ of $4 \mathrm{C} \mathrm{L}^{-1} \mathrm{~min}^{-1}$ and that oxidation reactions of other steel components such as $\mathrm{Mn}$ are not responsible for the low $F E$. This result suggests that locally available steel of unknown purity can be implemented in large scale EC systems without a loss of $F E$.

Figure 1 also compares Lab_04 to two experiments where another parameter than anode purity was varied: a higher CDR (Lab_54), and a different electrolyte without bicarbonate, silicate, and phosphate (oxyanion-free). In contrast to the $F E$ of Lab_04, the $F E$ of Lab_54 with a CDR of $54 \mathrm{C} \mathrm{L}^{-1} \mathrm{~min}^{-1}$ remained above 0.85 throughout the entire 20 runs. Remarkably, no differences in long-term $F E$ were observed between the different experiments at elevated $C D R$ $\left(15,32,54 \mathrm{C} \mathrm{L}^{-1} \mathrm{~min}^{-1}\right)$ and between the field and lab anode at high $C D R$ (data not shown). Furthermore, the same trend of sustained $F E>0.8$ was also observed at a $C D R$ of $4 \mathrm{C} \mathrm{L}^{-1} \mathrm{~min}^{-1}$ when oxyanions $(\mathrm{P}, \mathrm{Si}$, $\mathrm{HCO}_{3}$ ) were not present in the electrolyte. In all experiments with a high $F E$, the mass of iron deposits on the anode was consistently lower compared to Lab_04 and
Field_04, which suggests that the formation of anodic surface layers is correlated to the loss in $F E$ over time.

For the operation of EC systems in the field, the most significant result of this work is the strong impact of the $C D R$ on the $F E$ because the $C D R$ can be adjusted easily in Fe-EC using existing infrastructure. Because the performance of Fe-EC hinges on maintaining a high $F E$, operating EC systems at $\mathrm{CDR}>15 \mathrm{C} \mathrm{L}^{-1} \mathrm{~min}^{-1}$ can ensure efficient and reliable treatment. Furthermore, increasing $C D R$ also decreases total treatment time, which is beneficial in areas with intermittent electricity supply. Increasing the $C D R$ beyond $15 \mathrm{C} \mathrm{L}^{-1} \mathrm{~min}^{-1}$ had no observable effect on the $F E$ and is only recommended if shorter treatment times are required.

\section{CONCLUSIONS}

The aim of this study was to determine if long-term operation leads to a decrease in $F E$, a potential reason for the lower As removal efficiency observed for field EC-systems relative to those in the lab. Our results showed that the $F E$ continuously decreased over time under conditions similar to a system used for As removal in West Bengal, India. This drop in $F E$ should be addressed in the extended operation of Fe-EC systems in the field, possibly by increasing the electrolysis time incrementally over several months.

Our results also showed that the decrease in $F E$ was not caused by a low $\mathrm{Fe}$ anode purity. Instead, the electrolyte composition and the $C D R$ were identified as key parameters governing the $F E$. Applying a $C D R \geq 15 \mathrm{C} \mathrm{L}^{-1} \mathrm{~min}^{-1}$ or excluding oxyanions from the electrolyte improved the long-term $F E$. These modifications also correlated with surface layers of lower mass on the anode surface. Hence, a high and stable $F E$ in field systems can be achieved by increasing the applied current $(C D R)$.

\section{ACKNOWLEDGEMENTS}

CMvG acknowledges funding support by a NWO Veni grant (Project No. 14400).

\section{REFERENCES}

Amrose, S., Gadgil, A., Srinivasan, V., Kowolik, K., Muller, M., Huang, J. \& Kostecki, R. 2013. Arsenic removal from groundwater using iron electrocoagulation: effect of charge dosage rate. J. Environ. Sci. Health A 48(9): 1019-1030.

Amrose, S.E., Bandaru, S.R., Delaire, C., van Genuchten, C.M., Dutta, A., DebSarkar, A., Orr, C., Roy, J., Das, A. \& Gadgil, A.J. 2014. Electro-chemical arsenic remediation: field trials in West Bengal. Sci. Total Environ. 488: 539546.

APHA. 2015. Standard Methods for the Examination of Water \& Wastewater. American Public Health Association (APHA), Washington, DC, USA.

van Genuchten, C.M., Bandaru, S.R., Surorova, E., Amrose, S.E., Gadgil, A.J. \& Pena, J. 2016. Formation of macroscopic surface layers on $\mathrm{Fe}(0)$ electrocoagulation electrodes during an extended field trial of arsenic treatment. Chemosphere 153: 270-279. 